\title{
Solubility of Anthracene in Ternary Propanol + Butanol + Cyclohexane Solvent Mixtures
}

\author{
Taihe Deng and William E. Acree, J r.* \\ Department of Chemistry, University of North Texas, Denton, Texas 76203-5070
}

\begin{abstract}
Experimental solubilities are reported for anthracene dissolved in ternary 1-propanol + 1-butanol + cycl ohexane, 1-propanol + 2-butanol + cyclohexane, 2-propanol + 1-butanol + cyclohexane, and 2-propanol +2 -butanol + cyclohexane solvent mixtures at $298.15 \mathrm{~K}$. Nineteen compositions were studied for each of the four solvent systems. Results of these measurements are used to test the predictive ability of the ternary solvent form of the combined NIBS/Redlich-Kister equation. Computations showed that the model predicted the observed solubility behavior to within an overall average absolute deviation of about $1.6 \%$.
\end{abstract}

\section{Introduction}

Solid-liquid equilibrium data of organic nonelectrolyte systems are becoming increasingly important in the petroleum industry, particularly in light of present trends toward heavier feedstocks and the known carcinogenicity/ mutagenicity of many of the larger polycyclic aromatic compounds. Solubility data for a number of polycyclic aromatic hydrocarbons (i.e., anthracene and pyrene) and heteroatom polynuclear aromatics (i.e., carbazole, dibenzothiophene, and xanthene) have been published in the recent chemical literature. For a listing of references, see Acree (1994, 1995a,b). Despite efforts by experimentalists and scientific organizations, both in terms of new experimental measurements and critically evaluated data compilations, there still exist numerous systems for which solubility data are not readily available.

To address this problem, researchers have turned to group contribution methods and semiempirical equations to predict desired quantities from either pure component properties or measured binary data. In earlier studies we have used the binary solvent reduction of a predictive expression derived from a combined two- and three-body interactional mixing model as a mathematical representation for describing how the measured isothermal solubility of a crystalline solute varies with binary solvent composition. The binary reduction, referred to as the combined NIBS/Redlich-Kister equation, was found to accurately describe the observed solubility behavior in a large number of different binary solvent systems. We now extend our solubility studies to ternary solvent mixtures. Anthracene solubilities have been measured in the four ternary propanol + butanol + cyclohexane systems at $25^{\circ} \mathrm{C}$. Nineteen ternary compositions were studied for each of the four systems. Results of these measurements are used to test the predictive ability of expressions based upon the general mixing model used in deriving the combined NIBS/RedlichKister equation.

\section{Experimental Methods}

Anthracene (Acros, 99.9+ \%) was recrystallized three times from 2-propanone. 1-Propanol (Aldrich, 99+ \%,

\footnotetext{
* To whom correspondence should be addressed. E-mail: acree@unt.edu.
} FAX: (940) 565-4318. anhydrous), 2-propanol (Aldrich, 99+ \%, anhydrous), 1-butanol (Aldrich, HPLC, 99.8+ \%), 2-butanol (Aldrich, 99+ $\%$, anhydrous), and cyclohexane (Aldrich, HPLC, 99.9+ \%) were stored over molecular sieves and distilled shortly before use. Gas chromatographic analysis showed solvent purities to be $99.7 \mathrm{~mol} \%$ or better. Ternary solvent mixtures were prepared by mass so that compositions could be calculated to 0.0001 mole fraction. Karl Fischer titrations performed on select samples both prior to and after equilibration gave, to within experimental uncertainty, identical water contents (mass/mass \%) of $<0.01 \%$. The methods of sample equilibration and spectrophotometric analysis are discussed in an earlier paper (Powell et al., 1997). Experimental anthracene solubilities in the four propanol + butanol + cyclohexane solvent mixtures are listed in Table 1. Numerical values represent the average of between four and eight independent determinations, with the measured values being reproducible to within $\pm 1.5 \%$.

\section{Results and Discussion}

Expressions for predicting the thermodynamic properties of ternary nonelectrolyte systems have served as the point of departure for mathematical representation of experimental excess molar Gibbs energy, excess molar heat capacity, excess molar enthalpy, and excess molar volume data. Differences between predicted and observed values are expressed as

$$
\left(Z_{A B C}^{E}\right)^{\text {exp }}-\left(Z_{A B C}^{E}\right)^{\text {calc }}=x_{A} x_{B} x_{C} Q_{A B C}
$$

with Q-functions of varying complexity. For most systems encountered, the experimental data can be adequately represented by a power series expansion

$$
\begin{aligned}
Q_{A B C}=A_{A B C}+\sum_{i=1}^{r} B_{A B}^{(i)}\left(x_{A}-x_{B}\right)^{i}+ & \sum_{j=1}^{s} B_{A C}^{(j)}\left(x_{A}-x_{C}\right)^{j}+ \\
& \sum_{k=1}^{t} B_{B C}^{(k)}\left(x_{B}-x_{C}\right)^{k}
\end{aligned}
$$

though rarely are experimental data determined with sufficient precision tojustify more than a few parameters. 
Table 1. Experimental Mole Fraction Solubilities of Anthracene ( $x_{A}^{\text {sat }}$ ) in Ternary Propanol (B) + Butanol (C) + Cyclohexane (D) Solvent Mixtures at 298.15 K

\begin{tabular}{|c|c|c|c|c|c|}
\hline$x_{B}^{\circ}$ & $x_{c}^{\circ}$ & $x_{A}^{\text {sat }}$ & $x_{B}^{\circ}$ & $x_{C}^{\circ}$ & $x_{A}^{\text {sat }}$ \\
\hline \multicolumn{6}{|c|}{ 1-Propanol (B) + 1-Butanol (C) + Cyclohexane (D) } \\
\hline 0.4028 & 0.3242 & 0.00103 & 0.2050 & 0.1611 & 0.00147 \\
\hline 0.1855 & 0.6881 & 0.000908 & 0.7561 & 0.1317 & 0.000762 \\
\hline 0.3161 & 0.2543 & 0.00124 & 0.5720 & 0.2322 & 0.000898 \\
\hline 0.3021 & 0.4885 & 0.000962 & 0.1425 & 0.2171 & 0.00146 \\
\hline 0.7549 & 0.0932 & 0.000801 & 0.1260 & 0.7052 & 0.000961 \\
\hline 0.7477 & 0.1763 & 0.000722 & 0.2620 & 0.1061 & 0.001 \\
\hline 0.2323 & 0.6835 & 0.000845 & 0.1323 & 0.4225 & 0.001 \\
\hline 0.1248 & 0.5214 & 0.00117 & 0.4857 & 0.1009 & 0.001 \\
\hline 0.4545 & 0.4645 & 0.000792 & 0.5820 & 0.0966 & 0.00104 \\
\hline 0.5566 & 0.3649 & 0.000758 & & & \\
\hline \multicolumn{6}{|c|}{ 1-Propanol (B) + 2-Butanol (C) + Cyclohexane (D) } \\
\hline 0.3954 & 0.3274 & 0.000968 & 0.1987 & 0.1628 & 0.00144 \\
\hline 0.1829 & 0.6898 & 0.000 & 0.7537 & 0.1327 & 0.000722 \\
\hline 0.3100 & 0.2560 & 0.00118 & 0.5693 & 0.2327 & 0.000842 \\
\hline 0.2990 & 0.4892 & 0.000 & 0.1343 & 0.2183 & 0.00147 \\
\hline 0.7618 & 0.0907 & 0.00 & 0.1273 & 0.7061 & 0.00 \\
\hline 0.7512 & 0.1751 & 0.000676 & 0.2663 & 0.1078 & 0.00143 \\
\hline 0.2376 & 0.6800 & 0.000687 & 0.1323 & 0.4217 & 0.00122 \\
\hline & 0.5172 & 0.00106 & 0.4864 & 0.0989 & 0.00115 \\
\hline & 0.4628 & 0.000 & 0.5819 & 0.0959 & 0.00101 \\
\hline & & & & & \\
\hline \multicolumn{6}{|c|}{ 2-Propanol (B) + 1-Butanol (C) + Cyclohexane (D) } \\
\hline 0.3954 & 0.3278 & 0.000947 & 0.2010 & 0.1705 & 0.00143 \\
\hline 0.1837 & 0.6868 & 0.000865 & 0.7444 & 0.1414 & 0.000629 \\
\hline & & & & & 0.0 \\
\hline 002 & 0.49 & & 0.1469 & 0.2222 & 0.00 \\
\hline 0.7621 & 0.0887 & 0.000 & 0.1256 & 0.7022 & 0.000929 \\
\hline 0.7514 & 0.1725 & 0.000 & 0.2530 & 0.1066 & 0.00141 \\
\hline & & & 0.15 & 0.4067 & 0.00 \\
\hline & & & 0.48 & 0.1 & 0.00 \\
\hline 0.4555 & 0.4697 & 0.000700 & 0.5771 & 0.1016 & 0.000936 \\
\hline 0.5520 & 0.3711 & 0.000662 & & & \\
\hline \multicolumn{6}{|c|}{ 2-Propanol (B) + 2-Butanol (C) + Cyclohexane (D) } \\
\hline 0.3961 & 0.3220 & 0.000901 & 0.1891 & 0.1695 & 0.00141 \\
\hline & 0.706 & & 0.75 & 0.1 & 0.00 \\
\hline 0.2997 & 0.2619 & 0.001 & 0.5631 & 0.2387 & 0.000738 \\
\hline 0.2991 & 0.4964 & 0.000797 & 0.1236 & 0.2196 & 0.00144 \\
\hline 0.7598 & 0.0919 & 0.000641 & 0.1233 & 0.7068 & 0.000789 \\
\hline 0.7422 & 0.1829 & & 0.2564 & 0.1142 & 0.00138 \\
\hline 0.2324 & 0.6804 & & 0.1228 & 0.4273 & 0.00120 \\
\hline 0.1274 & 0.5218 & 0.00104 & 0.4746 & 0.1033 & 0.00106 \\
\hline 0.4458 & 0.4727 & 0.000599 & 0.5815 & 0.0972 & 0.000911 \\
\hline 0.5459 & 0.3711 & 0.000600 & & & \\
\hline
\end{tabular}

Conceptually, these ideas can be extended to solute solubilities in binary solvent mixtures; however, there has never been up until recently a sufficiently large solid solute solubility database to warrant computerized storage in equational form. With computerized data storage and retrieval becoming increasingly popular, it seems appropriate to discuss the various mathematical expressions that have been proposed in the chemical literature for describing the variation of solute solubility with binary solvent composition. Mathematical representations not only provide a means to screen experimental data sets for possible outliers in need of redetermination but also facilitate interpolation at solvent compositions falling between measured data points.

Acree and co-workers (Acree, 1992; Acree and Zvaigzne, 1991; Acree et al., 1991) suggested a possi ble mathematical representation for isothermal solubility data based upon the combined NIBS/Redlich-Kister model

$$
\begin{aligned}
\ln x_{A}^{\text {sat }}=x_{B}^{\circ} \ln \left(x_{A}^{\text {sat }}\right)_{B}+x_{C}^{\circ} \ln \left(x_{A}^{\text {sat }}\right)_{C}+ & \\
& x_{B}^{\circ} x_{C}^{\circ} \sum_{i=0}^{n} S_{i}\left(x_{B}^{\circ}-x_{C}^{\circ}\right)^{i}
\end{aligned}
$$

Table 2. Combined NIBS/Redlich-Kister Parameters Calculated from Anthracene Solubilities in the Sub-binary Solvent Systems

\begin{tabular}{cr}
\hline solvent (B) + solvent (C) & \multicolumn{1}{c}{$\mathrm{S}^{\mathrm{a}}$} \\
\hline 1-propanol (B) + 1-butanol (C) & 0.117 \\
& -0.015 \\
1-propanol (B) + cyclohexane (C) & 1.121 \\
& -0.040 \\
1-butanol (B) + cyclohexane (C) & 0.256 \\
& 0.741 \\
2-propanol (B) + 1-butanol (C) & -0.345 \\
2-propanol (B) + cyclohexane (C) & 0.223 \\
& 0.243 \\
& 0.011 \\
1-propanol (B) + 2-butanol (C) & 1.589 \\
2-propanol (B) + 2-butanol (C) & 0.143 \\
& 0.248 \\
2-butanol (B) + cyclohexane (C) & 0.000 \\
& 0.000 \\
& 0.097 \\
& 0.013 \\
& 1.260 \\
& -0.206
\end{tabular}

a Combined NIBS/Redlich-Kister curve-fit parameters are ordered as $\mathrm{S}_{0}, \mathrm{~S}_{1}$, and $\mathrm{S}_{2}$.

Table 3. Summarized Comparison between Observed Anthracene Solubilities in Ternary Propanol + Butanol + Cyclohexane Solvent Mixtures and Predicted Values Based upon the Combined NIBS/Redlich-Kister

\begin{tabular}{|c|c|}
\hline ternary solvent mixture & $\%$ Dev. $^{a}$ \\
\hline $\begin{array}{l}\text { 1-propanol (B) + 1-butanol (C) + cyclohexane (D) } \\
\text { 1-propanol (B) + 2-butanol (C) + cyclohexane (D) } \\
\text { 2-propanol (B) + 1-butanol (C) + cyclohexane (D) } \\
\text { 2-propanol (B) + 2-butanol (C) + cyclohexane (D) }\end{array}$ & $\begin{array}{l}1.45 \\
1.76 \\
1.70 \\
1.57\end{array}$ \\
\hline
\end{tabular}
Equation 4

where $x_{B}^{\circ}$ and $x_{C}^{\circ}$ refer to the initial mole fraction composition of the binary solvent calculated as if the solute were not present and $\left(x_{A}^{\text {sat }}\right)_{i}$ denotes the measured solute solubility in pure solvent $i$. The various $S_{i}$ "curve-fit" parameters can be evaluated via least-squares analysis. Published papers (Zvaigzne et al. 1993; Zvaigzne and Acree, 1994; Acree et al., 1994; Acree and Zvaigzne, 1994) have reported the calculated $S_{i}$ parameters for anthracene dissolved in the eight sub-binary solvent systems, as well as the measured mole fraction solubilities in 1-propanol $\left(x_{A}^{\text {sat }}=0.000591\right), 2$-propanol $\left(x_{A}^{\text {sat }}=0.000411\right), 1$-butanol $\left(x_{A}^{\text {sat }}=0.000801\right), 2$-butanol $\left(x_{A}^{\text {sat }}=0.000585\right)$, and cyclohexane $\left(x_{A}^{\text {sat }}=0.001553\right)$. Numerical values of the $S_{i}$ parameters have been tabulated in Table 2 for convenience.

Equation 3 expresses the "excess" logarithmic mole fraction solubility, relative to the simple $x_{B}^{\circ} \ln \left(x_{A}^{\text {sat }}\right)_{B}+x_{C}^{\circ}$ $\ln \left(x_{A}^{\text {sat }}\right)_{C}$ arithmetic average, in terms of the RedlichKister equation. For a ternary solvent system, the mathematical representation takes the form of

$$
\begin{array}{r}
\ln x_{A}^{\text {sat }}=x_{B}^{\circ} \ln \left(x_{A}^{\text {sat }}\right)_{B}+x_{C}^{\circ} \ln \left(x_{A}^{\text {sat }}\right)_{C}+x_{D}^{\circ} \ln \left(x_{A}^{\text {sat }}\right)_{D}+ \\
x_{B}^{\circ} x_{C}^{\circ} \sum_{i=0}^{r} S_{i, B C}\left(x_{B}^{\circ}-x_{C}^{\circ}\right)^{i}+x_{B}^{\circ} x_{D}^{\circ} \sum_{j=0}^{s} S_{j, B D}\left(x_{B}^{\circ}-x_{D}^{\circ}\right)^{j}+ \\
x_{C}^{\circ} x_{D}^{\circ} \sum_{k=0}^{t} S_{k, C D}\left(x_{C}^{\circ}-x_{D}^{\circ}\right)^{k}
\end{array}
$$

Equation 4 can be used to predict the solubility as a function of ternary solvent composition, provided that all of the various curve-fit parameters are known. The predic- 
1064 J ournal of Chemical and Engineering Data, Vol. 43, No. 6, 1998

tive ability of eq 4 is summarized in Table 3 for anthracene dissolved in the four propanol + butanol + cyclohexane systems. Examination of the numerical entries in Table 3 reveals that eq 4 predicts the solubility of anthracene to within an overall average absolute deviation of $1.62 \%$, which is comparable to the experimental uncertainty of $\pm 1.5 \%$. For the four systems studied, eq 4 was found to provide very accurate predictions of the observed sol ubility behavior.

\section{Literature Cited}

Acree, W. E., J r. Mathematical Representation of Thermodynamic Properties. Part 2. Derivation of the Combined Nearly I deal Binary Solvent (NIBS)/Redlich-Kister Mathematical Representation from a Two-Body and Three-Body Interactional Mixing Model. Thermochim. Acta 1992, 198, 71-79.

Acree, W. E., J r. Polycydlic Aromatic Hydrocarbons in Pureand Binary Solvents; IUPAC Solubility Data Series 54; Oxford University Press: Oxford, U.K., 1994.

Acree, W. E., J r. Polycyclic Aromatic Hydrocarbons: Binary Nonaque ous Systems: Part 1 (Solutes A-E); IUPAC Solubility Data Series 58; Oxford University Press: Oxford, U.K., 1995a.

Acree, W. E., J r. Polycyclic Aromatic Hydrocarbons: Binary Nonaque ous Systems: Part 2 (Solutes F-Z); IUPAC Solubility Data Series 59; Oxford University Press: Oxford, U.K., 1995b.

Acree, W. E., J r.; Zvaigzne, A. I. Thermodynamic Properties of Nonelectrolyte Solutions. Part 4. Estimation and Mathematical Representation of Solute Activity Coefficients and Solubilities in
Binary Solvents Using the NIBS and Modified Wilson Equations. Thermochim. Acta 1991, 178, 151-167.

Acree, W. E., J r.; Zvaigzne, A. I. Thermochemical Investigations of Hydrogen-Bonded Solutions. Part 5. Development of Predictive Equations for the Solubility of Anthracene in Binary Alcohol + Alcohol Mixtures Based Upon Mobile Order Theory. Fluid Phase Equilib. 1994, 99, 167-183.

Acree, W. E., J r.; McCargar, J. W.; Zvaigzne, A. I.; Teng, I.-L. Mathematical Representation of Thermodynamic Properties. Carbazole Solubilities in Binary Alkane + Dibutyl Ether and Alkane + Tetrahydropyran Solvent Mixtures. Phys. Chem. Liq. 1991, 23, 27-35.

Acree, W. E., J r.; Zvaigzne, A. I.; Tucker, S. A. Thermochemical Investigations of Hydrogen-Bonded Solutions: Development of a Predictive Equation for the Solubility of Anthracene in Binary Hydrocarbon + Alcohol Solvent Mixtures Based Upon Mobile Order Theory. Fluid Phase Equilib. 1994, 92, 233-253.

Powell, J. R.; Coym, K. S.; Acree, W. E., J r. Solubility of Anthracene in Binary Alcohol + 2-Methoxyethyl Ether Solvent Mixtures. J. Chem. Eng. Data 1997, 42, 395-397.

Zvaigzne, A. I.; Acree, W. E., J r. Solubility of Anthracene in Binary Alkane + 2-Butanol Solvent Mixtures. J. Chem. Eng. Data 1994, 39, 114-116.

Zvaigzne, A. I.; Teng, I.-L.; Martinez, E.; Trejo, J .; Acree, W. E., J r. Solubility of Anthracene in Binary Alkane + 1-Propanol and Alkane + 1-Butanol Solvent Mixtures. J . Chem. Eng. Data 1993, 38, 389392.

Received for review J une 23, 1998. Accepted September 2, 1998.

J 9801415 